\title{
Focused Ion Beam (FIB) and Energy Dispersive X-Ray (EDX) Analysis in Adhesive Dentistry
}

\author{
Lolita Rotkina $^{1,2}$, Christoph Zbaeren ${ }^{2}$, Joannis Katsoulis ${ }^{2,3}$, Tomohiro Takagaki ${ }^{4}$ and Markus Blatz ${ }^{2}$ \\ 1. Ioffe Physical-Technical Institute of Russian Academy of Sciences, St.Petersburg, Russia, \\ 2. University of Pennsylvania, School of Dental Medicine, Philadelphia, US, \\ 3. School of Dental Medicine, University of Bern, Bern, Switzerland, \\ 4. Tokyo Medical and Dental University, Tokyo, Japan.
}

The goal of the study was to analyze the bonding interface between Katana Zirconia and Panavia 2.0 using non-destructive technologies (FIB and EDX) in combination with conventional microscopy (SEM). Zirconium dioxide (zirconia) ceramics have become popular for dental restorations due to its excellent physical properties (high flexural strength), biocompatibility, and esthetics [1]. The bond strength of the luting agent to the zirconia material is an important factor for the long-term success of the restoration. To ensure a reliable bond between the zirconia restoration and the tooth, various cements and different surface treatments of the zirconia have been applied. In contrast to conventional methods, sample preparation with DualBeam Focused Ion Beam (FIB) technology, followed by Scanning Electron Microscopy (SEM) in Secondary (SE) and Backscattered (BSE) Imaging modes and Analytical Energy Dispersive X-Ray (EDX) mapping allows for visualization of this particular bonding interface without preparation artifacts, and may thus help to better understand the adhesion between the luting agent and zirconia material [2-4].

In this study, we systematically investigated the bonding interface between Katana Zirconia and Panavia 2.0. Zirconia surfaces were pre-treated with sandblasting (SND) with Alumina particles and Clearfil Ceramic Primer application (PRM). Four sets of samples (No-treatment, SND, PRM, SND+PRM) were cut with the FIB along the interface in order to examine the adherence of the materials to each other with high resolution SEM. Typical pictures were chosen to demonstrate how the sample preparation affects the bonding area (see Fig.1a). To access completely flat surface for detecting small amount of elements at interface, we performed FIB cut-outs of the interfacial areas under the mechanically polished surface (see Fig.1b). The EDX of the area with slight Backscattered contrast difference showed that some of the particles, which look like most of the cement ingredients, are trapped Alumina particles, which came from the sandblasting. The interface with double treatment was seamless and homogeneous with no evidence for big gaps between the zirconia and the cement (see Fig.2). The Backscattered Electron Image showed slight variation of the composition close to the interface (see Fig.3). The quantative mapping demonstrates it with no doubt (see Fig.4). Just like Panavia resin cement, Clearfil Ceramic Primer contains special phosphate monomers (MDP) that provide chemical bonds to metal oxides. Thus, the Zircona particles, which are resulting from the sandblasting process, and the blasting particles of Alumina, which are difficult to remove completely, become an integral part of the bonding interface. The primer with its high wettability covers each small gap in the sandblasted surface and surrounds of each particle. At the same time, it slightly dissolves the cement base and mixes the new adopted particles with the ones already in the cement. From High resolution SEM, the particles are almost nondistinguishable, and only the qualitative and quantative EDX of the areas, opened by the FIB, properly demonstrate the adhesive bonding interface. Our work is in in accordance with another recent study, which demonstrates no need for more treatment other than sandblasting and primer application, and compliments its results with the EDX mapping of the interface, showing its actual composition $[5,6]$. 


\section{References:}

[1] Markus B. Blatz, et al. Quintessence Int 2007;38:745-753

[2] Van Meerbeek B, et al, Dent Mater. 1995 Mar;11(2):87-92

[3] Giannuzzi, Lucille A. et al. Journal of Oral and Maxillofacial Surgery, Volume 65, Issue 4, 737 - 747

[4] M. Inokoshi, et al, J Dent Res 93(4):329-334,

[5] Asuka et al, Dental Materials Journal 2016; 35(1): 29-36

[6] We acknowledge the access to the major instruments used for the experimental work it this study: FEI Strata DB FIB and FEI Quanta 600 ESEM with BSED at the Nanoscale Characterization Facility of the University of Pennsylvania, and FEI Quanta 400 ESEM with an Oxford INCA EDS in the CMIRT at West Chester University. Special acknowledgement goes to Dr. Fred Monson for his advising and encouragement in many ways.
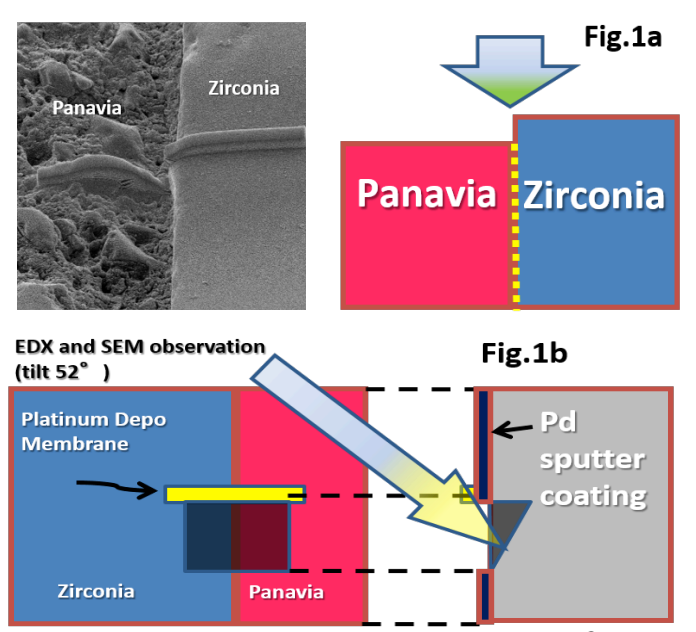

Fig.2
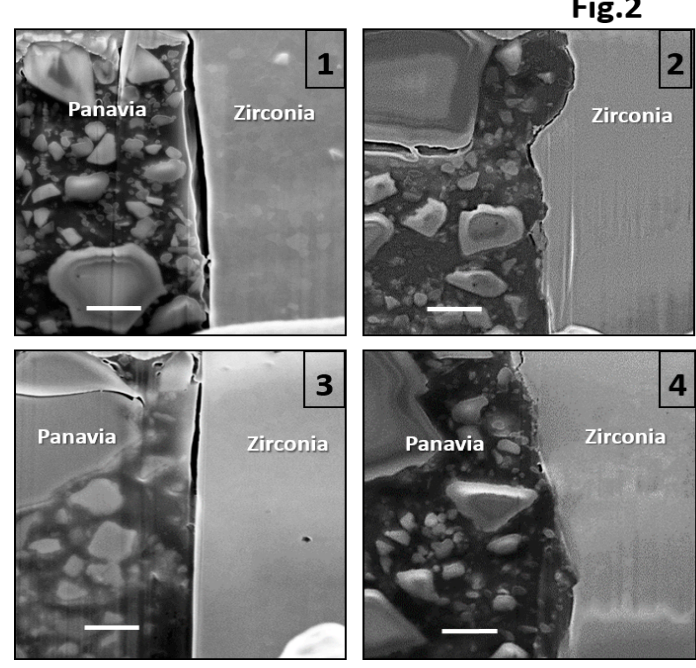

Fig 1. a) Mechanical polishing create altitude gap between zirconia and cement; b) FIB cross-sectioning diagram for accessing deep interfacial areas for SEM and EDX analysis.

Fig 2. Typical interfaces: 1 -no treatment of the Zirconia; $2-$ Alumina sandblast treated; 3 - treated with the Clearfil Ceramic Primer; 4 - Alumina sandblasted and Clearfil Ceramic treated. The scale bar is $1 \mu \mathrm{m}$.

Fig 3. Backscattered Electron Image, the interface in pseudo colors.

Fig 4. EDX map collected from the area with seamless interface and slight difference in the backscattered contrast reveals the presence of Alumina particles, which are completely embedded in the cement.
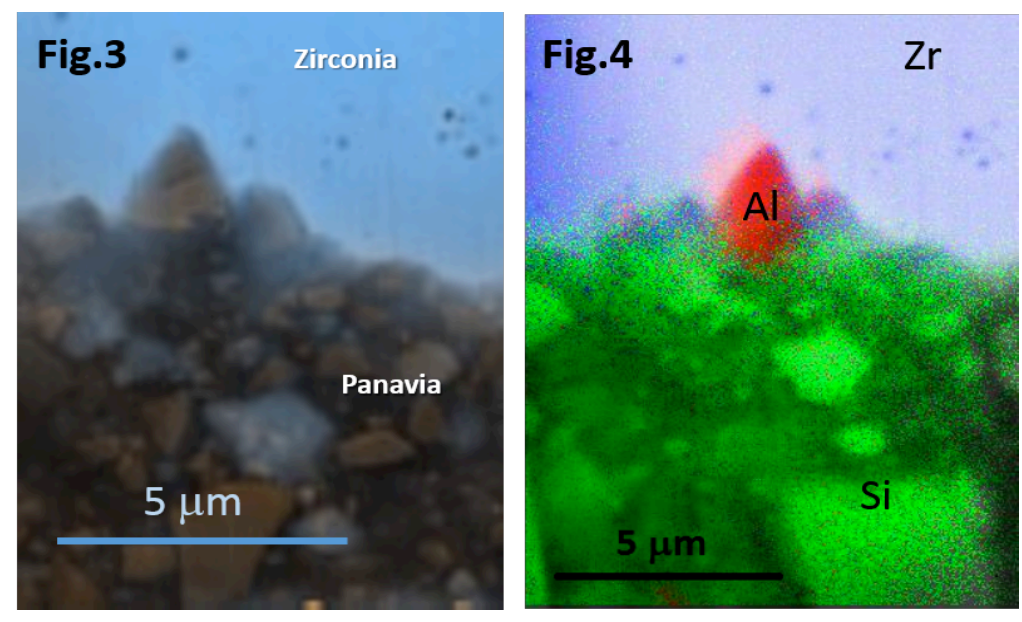